\title{
Controllable magnetic thermal rectification in a SMM dimmer with the Dzyaloshinskii-Moriya interaction
}

\author{
Ai-Hua Xu ${ }^{1}$, Juan Liu ${ }^{2}$,Tian Ju-Ping ${ }^{3}$ and Bo Luo ${ }^{2,3 *}$
}

1. School of Marxism, China University of Geosciences, Wuhan, 430074 Hubei, People's Republic of China.

2. Wuhan national High Magnetic Field Center, Academy of electronic and electrical engineering and School of Physics, Huazhong University of Science and Technology, Wuhan 430074, People's Republic of China.

3. School of Mechanical and Electrical, Changjiang Polytechnic, Wuhan 430074, People's Republic of China.

* Corresponding author: lb_hust_phys@hust.edu.cn

PACS: $05.60 . \mathrm{Gg}$

PACS: $66.70 . \mathrm{Lm}$

PACS: $74.25 . \mathrm{fg}$

\begin{abstract}
Using the quantum master equation, we studied the thermally driven magnonic spin current in a single-molecule magnet (SMM) dimer with the Dzyaloshinskii-Moriya interaction (DMI). Due to the asymmetric DMI, one can observe the thermal rectifying effect in the case of the spatial symmetry coupling with the thermal reservoirs. The properties of the thermal rectification can be controlled by tuning the angle and intensity of the magnetic field. Specially, when the DM vector and magnetic field point at the specific angles, the thermal rectifying effect disappears. And this phenomenon does not depend on the intensities of DMI and magnetic field, the temperature bias and the magnetic anisotropies of the SMM.
\end{abstract}




\section{I . Introduction}

Single-molecule magnet (SMM) is a nanomagnetic material. It has attracted much attention due to the potential applications and novel quantum phenomenons, e.g. molecule-based spintronics devices, information storage, quantum information processing ${ }^{1-5}$ and so on. At present, the thermally driven current (eg. spin, charge and thermal current) is a fascinating topic in nanotechnology due to the thermoelectricity providing an opening for a sustainable energy source $^{6}$. Many interesting results have been obtained such as thermal spin valves ${ }^{7}$, thermal spin-transfer torque ${ }^{8}$, ultrahigh spin thermopower ${ }^{9}$, gate-voltage-dependent oscillations of charge thermopower and pure spin current ${ }^{10,11}$. Notably, the thermally driven current can be carried not only by phonons and electrons but also by magnetons (spinons) in the magnetic material ${ }^{12-16}$.

The antisymmetric Dzyaloshinskii-Moriya interaction (DMI) is met in the most of SMMs and plays a key role in physical properties of molecules. Due to the DMI broken spin and spatial symmetry, the characteristic electron transport signatures have been studied, for example, a specific magnetic field dependence of the transport, nonlinear transport signatures, the violation of spin selection rules ${ }^{17-21}$. In experiment, DMI has been found in the Mn6, Mn12 and $\mathrm{V}^{22}, \mathrm{~V} 15$-based molecular ${ }^{23,24}$. Therefore, looking for the role of the DMI under the nonequilibrium conditions is crucial to modulating magnet thermal transfer.

In this paper, we investigated the magnetic thermal transfer in a SMM dimer with the Dzyaloshinskii-Moriya interaction (DMI), which was coupling with two boson thermal reservoirs. Using the quantum master equation, the influences of Dzyaloshinskii-Moriya on the magnetic thermal transport were discussed in detail. In the case of the spatial symmetric coupling with thermal baths, DMI can induce the thermal rectification effect due to the spatial inversion symmetry broken. For a given DM vector, the direction and efficiency of magnetic thermal rectification can be modified by tuning the direction and the intensity of the external magnetic field. Moreover, when the DM vector and magnetic field point at the specific direction, the thermal rectifying effect disappears. Specially, this phenomena does not depend on the intensity of DMI and magnetic field, the temperature bias and the magnetic anisotropies of SMM.

\section{Calculation Method}

The general Hamiltonian is expressed as 


$$
H=H_{S M M}+\sum_{v=L, R} H_{v}+V_{v}
$$

The first term $H_{S M M}=H_{i s}+H_{e x}$ describes an isolated SMM dimmer, in which the $H_{i s}$ and $H_{e x}$ is written as, respectively

$$
\begin{aligned}
& H_{i s}=\sum_{i=1,2}-D_{i}^{l}\left(S_{i}^{z}\right)^{2}+E_{i}^{t}\left(\left(S_{i}^{x}\right)^{2}-\left(S_{i}^{y}\right)^{2}\right)-g \mu_{B} \vec{h} \cdot \vec{S}_{i} \\
& H_{e x}=J \vec{S}_{1} \cdot \vec{S}_{2}+\vec{D} \cdot(\vec{S} \times \vec{S})
\end{aligned}
$$

Where, $H_{i s}$ describes the two isolated SMMs and $H_{e x}$ denotes the exchange interaction between these two. $D_{i}^{l}$ and $E_{i}^{t}$ are longitudinal and transverse anisotropic parameters of the i-th SMM $(i=1,2)$, respectively. $\vec{h}$ is the external magnetic field and $g=2$ is the Lander factor. $\mu_{B}$ is Bohr magneton. $\vec{S}_{i}$ is the spin vector. $J$ denotes the strength of Heisenberg interaction. $\vec{D}$ is the Dzyaloshinskii-Moriya vector. $H_{v}(v=L, R)$ is an isolated boson thermal reservoir with the temperature $T_{v}$. The Hamiltonian can be expressed as:

$$
H_{v}=\sum_{i} \omega_{i} \dot{a}_{, v} a
$$

where $a_{v, i}^{\dagger}\left(a_{v, i}\right)$ is the creation(annihilation) operator of bosons with the harmonic frequency $\omega_{i}$ in the $v$ thermal reservoir. The spectral density of the $v$ thermal bath is a continuous function of $\omega$, which is expressed as $^{25}$

$$
\rho(\omega)=\left\{\begin{array}{cc}
\omega_{c}^{1-s} e^{-\frac{\omega}{\omega_{c}}} \omega^{s} & \omega>0 \\
0 & \omega \leq 0
\end{array}\right.
$$

Here $\omega_{c}$ denotes the cutoff-frequency and $\rho(\omega)$ almost equals to zero in the high-frequency. The parameter s determines the low-frequency behavior of $\rho(\omega)$, which looks like a linear in the low-frequency with $s=1$.

The operator $V_{v}(v=L, R)$ is the system-lead interaction and we can use the following bipartite form:

$$
V_{v}=\lambda_{v} B_{v} S
$$


where $S_{v}$ is a subsystem operator $S_{v} \in\left(S_{x}, S_{y}, S_{z}\right)$ and $B_{v}$ is a bath operator which depends on the choices of a bath Hamiltonian. In this paper, we set $S_{L}=S_{R}=S_{x}$ and $B_{v}=\sum_{i}\left(a_{v, i}^{\dagger}+a_{v, i}\right)$.

In the weak coupling limit and under the Born-Markov approximation, the steady-state thermal current is obtained as ${ }^{26,27}$

$$
I=\frac{1}{2} \sum_{m, n} E_{m, n} P_{n}\left[\left|S_{m, n}^{L}\right|^{2} k_{n \rightarrow m}^{L}\left(T_{L}\right)-\left|S_{m, n}^{R}\right|^{2} k_{n \rightarrow m}^{R}\left(T_{R}\right)\right]
$$

where $P_{n}$ is the solution of the populations $P_{n}(t)=\operatorname{Tr}_{B}\left[\rho_{n, n}(t)\right]$ under the steady-state and the normalization conditions and $P_{n}(t)$ satisfies the equation 28,29

$$
\dot{P}_{n}(t)=\sum_{v, m}\left|S_{m, n}^{v}\right|^{2} P_{m}(t) k_{m \rightarrow n}^{v}\left(T_{v}\right)-P_{n}(t) \sum_{v, m}\left|S_{m, n}^{v}\right|^{2} k_{n \rightarrow m}^{v}\left(T_{v}\right)
$$

$k_{m \rightarrow n}^{v}$ is the Fermi golden rule transition and expressed as

$$
k_{n \rightarrow m}^{v}\left(T_{v}\right)=\lambda_{v}^{2} \int_{-\infty}^{\infty} d \tau e^{i E_{n, m} \tau}\left\langle B_{v}(\tau) B_{v}(0)\right\rangle_{T_{v}}
$$

where $E_{n, m}=E_{n}-E_{m}$ is the energy difference and $B_{v}(\tau)=e^{i H_{v} \tau} B_{v} e^{-i H_{v} \tau}$ is a operator in interaction picture. $\langle O\rangle_{T_{v}}$ means the thermal average value.

\section{Result and Discussion}

In this paper, $\mathrm{J}$ is taken to be an energy unit $(J=1) . \omega_{c}=100$ and $\lambda_{L}=\lambda_{R}=1$. Traditionally, the direction of $\mathrm{SMM} \operatorname{spin}\left(S_{z}\right)$ is alone with z-axis. The two vectors $(\vec{h}$ and $\vec{D})$ are denoted by spherical coordinates, namely, $\vec{h}=\left(R_{h}, \theta_{h}, \phi_{h}\right)$ and $\vec{D}=\left(R_{D}, \theta_{D}, \phi_{D}\right)$, in which $R, \theta(\in[0, \pi))$ and $\phi(\in[0,2 \pi))$ gives the modulus of the vector, polar angle and azimuthal angle respectively. $T_{R}\left(T_{L}\right)$ denotes the temperature of the right lead (left lead). $T_{a}=\left(T_{R}+T_{L}\right) / 2$ is the average temperature parameter and $\Delta T=T_{L}-T_{R}$ is the temperature difference. $\eta=\left|I^{+} / I^{-}\right|$is the rectification efficiency, in which $I^{+}$is the current in the positive temperature difference region $\left(T_{L}-T_{R}>0\right)$ and $I^{-}$is the current in the negative temperature difference region $\left(T_{L}-T_{R}<0\right)$. We choose the isolated molecular spin is equal to $1(S=1)$. Taking into account the $E_{1(2)}^{t}$ is much small than the $D_{1(2)}^{l}$, we can neglect the effects of the $E_{1(2)}^{t}$. Obviously, the magnetic 
field and transverse anisotropic $\left(D_{1}^{l}=D_{2}^{l}\right)$ can not give rise to the negative differential thermal conductance or thermal rectification effect because both of them can not break the spatial symmetry.

At first, we considered the influences of the longitudinal anisotropy and DMI on the magnetic thermal current without the external magnetic field, respectively. We choose the direction of DMI parallel with the longitudinal anisotropy $\left(\vec{D}=\left(R_{D}, 0,0\right)\right)$. From figure.1 (a) and (b), one can find that the thermal currents increase firstly, and then decrease with the increase of the intensities of $D_{1}^{l}\left(D_{2}^{l}\right)$ and $R_{D}$ at the fixed temperature difference. Comparing with the insets in figure.1 (a) and (b), it is clearly shown that the thermal currents are not symmetry about $D_{1}^{l}\left(D_{2}^{l}\right)=0$ without DMI, but are symmetry about $R_{D}=0$ without $D_{1}^{l}$ and $D_{2}^{l}$. When the type of Heisenberg interaction is changed, the magnetic thermal current does not change with $D_{1}^{l}\left(D_{2}^{l}\right)$, however the change of the magnetic thermal current occurs with $R_{D}$. These phenomena are due to the difference influences of the longitudinal anisotropy $D_{1}^{l}\left(D_{2}^{l}\right)$ and DMI on the eigen-energies of the system ${ }^{17,21}$.

Following, we considered the influences of DM vector on the thermal rectification effect without the external magnetic field. Here, we set the longitudinal anisotropy by a fixed value $D_{1}^{l}\left(D_{2}^{l}\right)=5$. In figure.2 (a), we show the rectification efficiency $\eta=\left|I^{+} / I^{-}\right|$as the function of the function of $\theta_{D}$ and $\phi_{D}$ with $R_{D}=1$ and $\Delta T=7.92$. We can find $\eta$ satisfies the symmetry $\eta\left(\theta_{D}, \phi_{D}\right)=\eta\left(\pi-\theta_{D}, \pi+\phi_{D}\right)$. Meanwhile, we also observed that the direction and intensity of the rectification efficiency are strongly related to the values of $\theta_{D}$ and $\phi_{D}$. Specially, the thermal rectification disappears $(\eta=1)$ when the angle of the vector DM is $\left(\frac{m}{2} \pi, \phi_{D}\right)$ or $\left(\theta_{D}, \frac{n}{2} \pi\right)(m=[0,1], n=[0,1,2,3])$. In figure.2 (b), we showed the rectification efficiency $\eta=\left|I^{+} / I^{-}\right|$as the function of $R_{D}$ and $\Delta T$ with $T_{a}=4, \theta_{D}=\pi / 3$ and $\phi_{D}=\pi / 4$. Observably, the $R_{D}$ can also modify the direction and intensity of $\eta$. It was interesting that rectification efficiency $\eta=\left|I^{+} / I^{-}\right|$does not always increases monotonically with increasing the 
temperature difference $\Delta T$. In detail, we plotted the $\eta$ as the function of $R_{D}$ and $\Delta T$ with the series of $\left(\theta_{D}, \phi_{D}\right)$ in figure.2(c) and (d), respectively. One can find that the $\eta$ is satisfied with the identical equation $\eta\left(R_{D}, \theta_{D}, \phi_{D}\right) \times \eta\left(-R_{D}, \theta_{D}, \phi_{D}\right) \equiv 1$ and the maximum (minimum) $\eta$ is strongly dependent on the values of $R_{D}, \theta_{D}$ and $\phi_{D}$. From figure.2(b) and (d), the non-monotonic increase of $\eta$ with the increasing of $\Delta T$ is related to the values of $R_{D}, \theta_{D}$ and $\phi_{D}$ with the fixed average temperature. Specially, the rectification efficiency $\eta$ is a constant equal to 1 when $\theta_{D}=\frac{m}{2} \pi(m=0,1)$ or $\phi_{D}=\frac{n}{2} \pi(n=0,1,2,3)$, and this phenomenon is independent on the value of $R_{D}$ and $\Delta T$ in figure. 2 (c) and (d).

Finally, we investigated the external magnetic field affects on the thermal rectification efficiency $\eta$ for a given DMI vector. In figure.3 (a) and (b), we displayed the $\eta$ as the function of $\left(\theta_{h}, \phi_{h}\right)$ and $\left(R_{h}, \phi_{h}\right)$ with $\vec{D}=\left(3.2, \frac{\pi}{3}, \frac{\pi}{6}\right), D_{1}^{l}\left(D_{2}^{l}\right)=5$ and $\Delta T=7.92$, respectively. It is observed that $\eta$ satisfies the symmetry $\eta\left(\theta_{h}, \phi_{h}\right)=\eta\left(\pi-\theta_{h}, \pi+\phi_{h}\right)$ and significantly enhanced by tuning the angle and strength of magnetic field. Meanwhile, the direction of thermal rectification can also controlled by tuning the angle and strength of magnetic field. Figure.3(c)and(d) show the $\eta$ as the function of $R_{h}$ and $\Delta T$ with the series of the vector DM, magnetic field $\vec{h}$ and $D_{1}^{l}\left(D_{2}^{l}\right)$, respectively. Interestingly, when the $\vec{h}$ and $\vec{D}$ are satisfied with $\vec{h}\left(R_{h}, \frac{\pi}{2}, \varphi_{h}\right)$ and $\vec{D}\left(R_{D}, \frac{\pi}{2}, \varphi_{D}\right)$, in which $\varphi_{h}$ and $\varphi_{D}$ are arbitrary values not necessary equal to each other, or $\vec{h}\left(R_{h}, \frac{\pi}{2}, \frac{n}{2} \pi\right)$ and $\vec{D}\left(R_{D}, \theta_{D}, \frac{m}{2} \pi\right)(n, m=0,1,2,3)$, the thermal rectification efficiency $\eta$ is constant equal to 1 , which is not depend on the values of $R_{h}$ and $\Delta T$. Similarly, one can observe that the $\eta$ first increase, then decrease with the increasing of $\Delta T$ in figure.3 (d) (green line). 
We studied the magnetic thermal current in a SMM dimer with the Dzyaloshinskii-Moriya interaction. The results showed Dzyaloshinskii-Moriya interaction played the crucial role of generating the thermal rectification effect due to its anti-symmetric. The features of magnetic thermal rectification are determined by tuning Dzyaloshinskii-Moriya vector and the external magnetic field. Specially, the angles of the magnetic field and DMI are more important than the strengths of them. We expected the similar phenomena can be observed in other spin systems with Dzyaloshinskii-Moriya interaction and all these phenomena might be useful for designing thermoelectricity molecular devices based on the magnetism.

\section{Acknowledgments}

This work was supported by China Postdoctoral Science Foundation funded project No. 0108012012 and the National Science Foundation of China under Grants No.11404121. Numerical calculations presented in this paper were carried out using the High Performance Computing Center experimental tested in SCTS/CGCL (see http://grid.hust.edu.cn/hpcc).

\section{References}

G. Christou, D. Gatteschi, D. N. Hendrickson, and R. Sessoli, Mrs Bulletin 25, 66 (2000).

A. Ardavan, O. Rival, J. J. Morton, S. J. Blundell, A. M. Tyryshkin, G. A. Timco, and R. E. Winpenny, Physical review letters 98, 057201 (2007).

M. Evangelisti and E. K. Brechin, Dalton transactions 39, 4672 (2010).

R. E. Winpenny, Angewandte Chemie International Edition 47, 7992 (2008).

M. N. Leuenberger and D. Loss, Nature 410, 789 (2001).

M. S. Dresselhaus, G. Chen, M. Y. Tang, R. Yang, H. Lee, D. Wang, Z. Ren, J. P. Fleurial, and P. Gogna, Advanced materials 19, 1043 (2007).

B. Sales, R. Jin, and D. Mandrus, 2004).

Z. Zhang, L. Jiang, R. Wang, B. Wang, and D. Xing, Applied Physics Letters 99, 133110 (2011).

B. Luo, J. Liu, J.-T. Lü, J.-H. Gao, and K.-L. Yao, Scientific reports 4 (2014).

R.-Q. Wang, L. Sheng, R. Shen, B. Wang, and D. Xing, Physical review letters 105, 057202 (2010).

Z. Zhang, L. Jiang, R. Wang, B. Wang, and D. Xing, Applied Physics Letters 97, 242101 (2010).

A. Palacio-Morales, A. Pourret, G. Knebel, T. Combier, D. Aoki, H. Harima, and J. Flouquet, Physical Review Letters 110 (2013).

A. Sologubenko, K. Gianno, H. Ott, U. Ammerahl, and A. Revcolevschi, Physical review letters 84, 2714 (2000).

C. Hess, H. ElHaes, B. Büchner, U. Ammerahl, M. Hücker, and A. Revcolevschi, Physical review letters 93, 027005 (2004).

N. Hlubek, P. Ribeiro, R. Saint-Martin, A. Revcolevschi, G. Roth, G. Behr, B. Büchner, and C. Hess, Physical Review B 81, 020405 (2010). 
J. Thingna and J.-S. Wang, EPL (Europhysics Letters) 104, 37006 (2013).

S. Herzog and M. R. Wegewijs, Nanotechnology 21, 274010 (2010).

M. Pletyukhov, D. Schuricht, and H. Schoeller, Physical review letters 104, 106801 (2010).

C. Romeike, M. Wegewijs, and H. Schoeller, Physical review letters 96, 196805 (2006).

G. González and M. N. Leuenberger, Physical review letters 98, 256804 (2007).

B. Luo, J. Liu, and K.-L. Yao, Physics Letters A 377, 2428 (2013).

M. I. Belinsky, Physical Review B 84 (2011).

M. Machida, T. Iitaka, and S. Miyashita, Physical Review B 86 (2012).

N. Konstantinidis and D. Coffey, Physical Review B 66, 174426 (2002).

H. Wichterich, M. J. Henrich, H.-P. Breuer, J. Gemmer, and M. Michel, Physical Review E 76, 031115 (2007).

L.-A. Wu, X. Y. Claire, and D. Segal, Physical Review E 80, 041103 (2009).

N. Zeng and J.-S. Wang, Physical Review B 78, 024305 (2008).

L.-A. Wu and D. Segal, Physical Review A 84, 012319 (2011).

W. H. Zurek, Reviews of Modern Physics 75, 715 (2003).
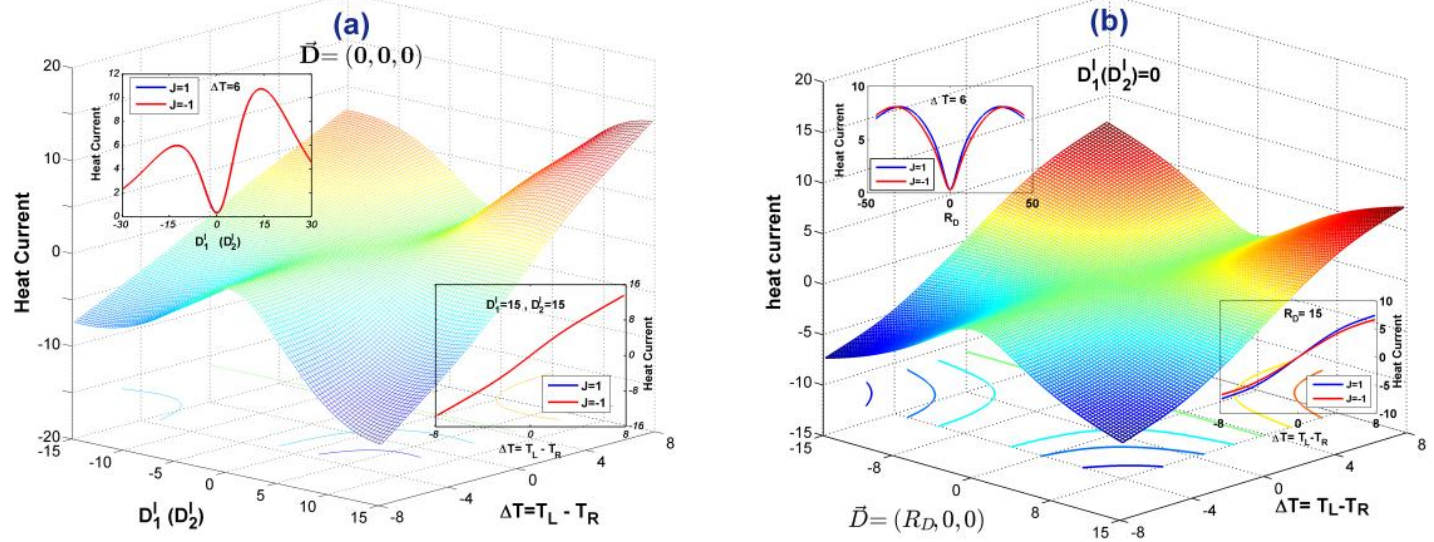

Figure.1 (a) and (b) displays the thermal current as the function of $D_{1}^{l}\left(D_{2}^{l}\right)$ and $R_{D}$ respectively without the external magnetic field. The upper-left insets in (a) and (b) display the thermal current as the function of the intensity of $D_{1}^{l}\left(D_{2}^{l}\right)$ and $\vec{D}$ at the fixed temperature difference $\Delta T=6$, respectively. The lower-right corners in (a) and (b) show the thermal current as the function of temperature difference $\Delta T$ at the fixed $D_{1}^{l}\left(D_{2}^{l}\right)=15$ and $R_{D}=15$, respectively. Others physics parameters are set as follow: the molecular spin is equal to 1 ; the average temperature $T_{a}=4$ is fixed and the temperatures of the two baths are set as $T_{L}=T_{a}+\Delta T / 2$ and $T_{R}=T_{a}-\Delta T / 2$ respectively. The transverse anisotropy is $\left(E_{1}^{t}, E_{2}^{t}\right)=(0,0)$. The bosonic baths are adopted with the $\omega_{c}=100$ and $s=1$. 

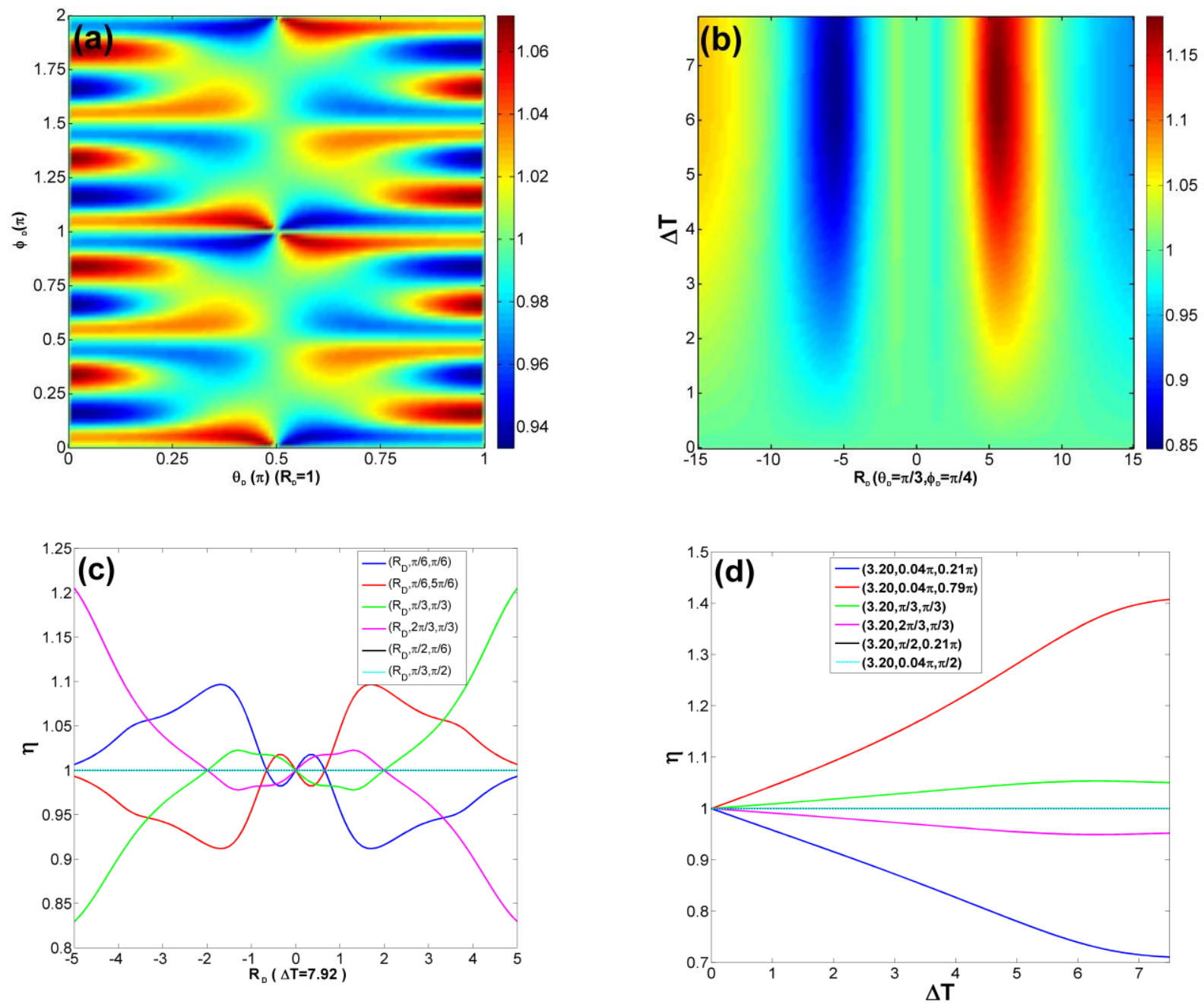

Figure.2. (a) displays the $\eta$ as the function of $\theta_{D}$ and $\phi_{D}$ with $R_{D}=1$ and $\Delta T=7.92$. (b) shows the $\eta$ as the function of $R_{D}$ and $\Delta T$ with $\theta_{D}=\frac{\pi}{3}$ and $\phi_{D}=\frac{\pi}{4}$. Panels (c) and (d) display the $\eta$ as the function of $R_{D}$ and $\Delta T$ with the series of $\theta_{D}$ and $\phi_{D}$, respectively. The magnetic field is set as $\vec{h}=(0,0,0)$ and the longitudinal anisotropy is $D_{1}^{l}\left(D_{2}^{l}\right)=5$. The other parameters are set the same as in Figure.1. 

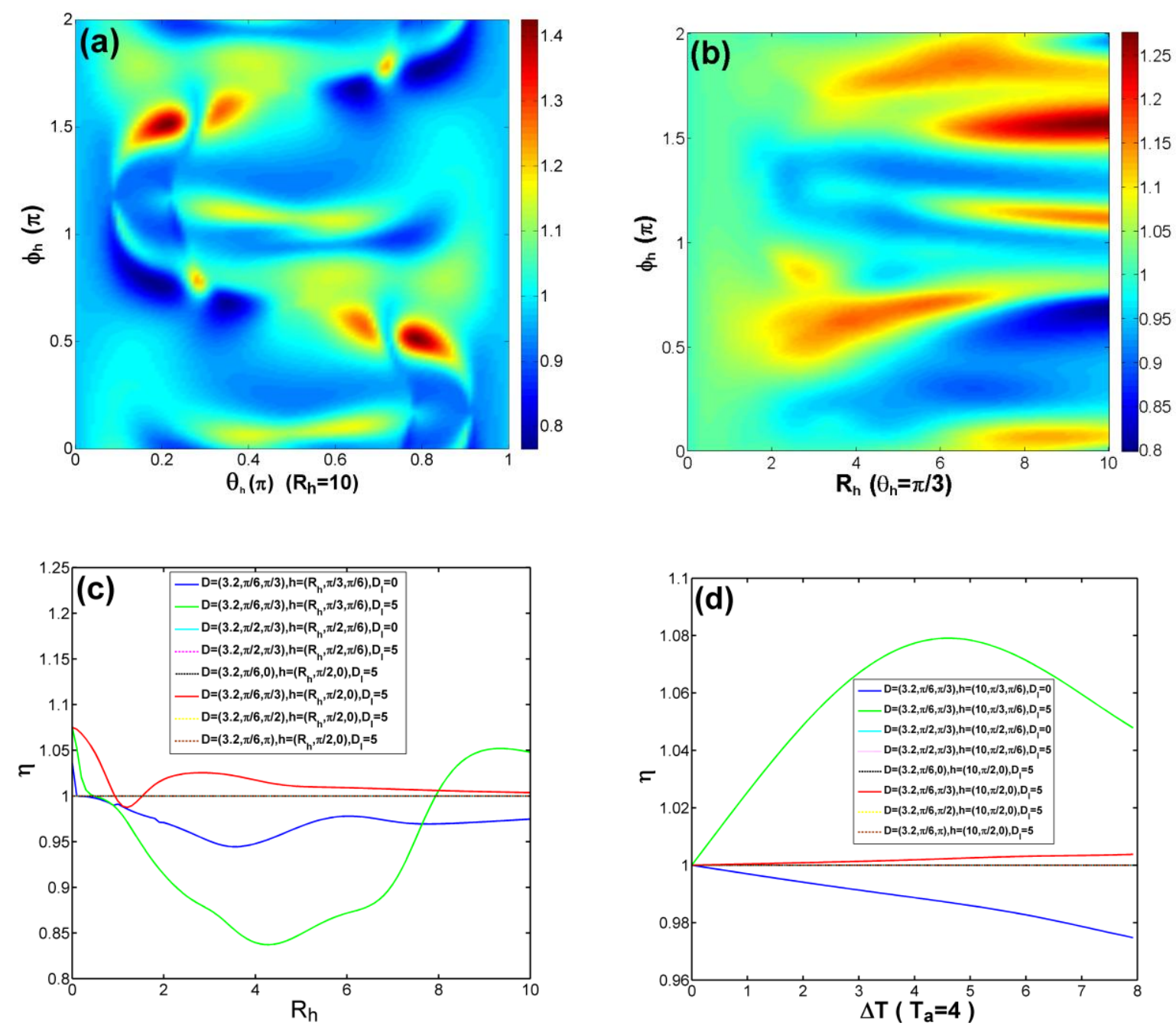

Figure.3. (a) and (b) display the $\eta$ as the function of $\left(\theta_{h}, \phi_{h}\right)$ and $\left(R_{h}, \phi_{h}\right)$ with $\vec{D}=\left(3.2, \frac{\pi}{3}, \frac{\pi}{6}\right)$, $D_{1}^{l}\left(D_{2}^{l}\right)=5$ and $\Delta T=7.92$, respectively. Panels (c) and (d) show the $\eta$ as the function of $R_{h}$ and $\Delta T$ with the series of the vector DM, magnetic field $\vec{h}$ and $D_{1}^{l}\left(D_{2}^{l}\right)$, respectively. The other parameters are set the same as in Figure.1. 